\title{
Subjective Health Status: An Easily Available, Independent, Robust And Significant Predictive Factor At The Prometaphase of Vaccination Programs For The Vaccination Behavior of Chinese Adults
}

Zuxing Wang ( $\sim$ zuxingwang7090@163.com )

Sichuan Provincial Center for Mental Health

Lili Chen

Sichuan Provincial Center for Mental Health

Jun Xiao

Sichuan Provincial Center for Mental Health

Fugui Jiang

Sichuan Provincial Center for Mental Health

Wenjiao Min

Sichuan Provincial Center for Mental Health

Shuyun Liu

Wenjiang District People's Hospital

Yunqiong Wang

Sichuan Provincial Center for Mental Health

Mengsha Qi

Wenjiang District People's Hospital

\section{Research Article}

Keywords: COVID-19, vaccination behavior, vaccination rate, subjective health status, related factors

Posted Date: November 23rd, 2021

DOI: https://doi.org/10.21203/rs.3.rs-1033041/v1

License: (c) (i) This work is licensed under a Creative Commons Attribution 4.0 International License.

Read Full License 
Version of Record: A version of this preprint was published at BMC Psychiatry on March 14th, 2022. See the published version at https://doi.org/10.1186/s12888-022-03830-5. 
Subjective health status: an easily available, independent, robust and significant predictive factor at the prometaphase of vaccination programs for the vaccination behavior of Chinese adults

Zuxing Wang ${ }^{1,2 *, \#}$, Lili Chen ${ }^{1,2 \#}$, Jun Xiao ${ }^{1,2}$, Fugui Jiang ${ }^{1,2}$, Wenjiao Min ${ }^{1,2}$, Shuyun Liu ${ }^{3}$, Yunqiong Wang ${ }^{1,2}$, Mengsha $\mathrm{Qi}^{3,4 *}$

1. Sichuan Provincial Center for Mental Health, Sichuan Academy of Medical Sciences \& Sichuan Provincial People's Hospital, Chengdu, 610072, China.

2. Key Laboratory of psychosomatic medicine,Chinese Academy of Medical Sciences, Chengdu 610072, China.

3. The ministry of science and education, Wenjiang District People's Hospital, Chengdu, 611130, China.

4. Department of Psychology, Chengdu Medical College, Chengdu, 610500, China.

\#Zuxing Wang and Lili Chen contributed equally.

*Correspondence: Mengsha Qi (1666824742@qq.com) and Zuxing Wang (zuxingwang7090@163.com) at Sichuan Provincial Center for Mental Health, Sichuan Academy of Medical Sciences \& Sichuan Provincial People's Hospital and Key Laboratory of psychosomatic medicine, Chinese Academy of Medical Sciences, Chengdu 610072, China.

Running title: Subjective health status and vaccination behavior

Number of words in the abstract: 222

Number of words in the text: 3730

Number of tables: 3

Number of figure: 1 
Background. The World Health Organization (WHO) proposed COVID-19 vaccination as an emergent and important method to end the COVID-19 pandemic. Since China started vaccination programs in December 2020, vaccination has spread to provinces and municipalities nationwide. Previous research has focused on people's vaccination willingness and its influencing factors but has not examined vaccination behavior. We examine the effectiveness of psychosocial factors in predicting vaccination behavior.

Methods. A cross-sectional online survey was performed among Chinese adults on 8 May and 4 June 2021. The statistical analysis of the data included univariate analysis, receiver operator characteristics (ROC) analysis and ordinal multiclassification logistic regression model analysis.

Results. Of the 1300 respondents, 761 (58.5\%) were vaccinated. Univariate analysis showed that a high education level and good subjective health status were protective factors for vaccination behavior, while suffering from chronic diseases was a risk factor. ROC analysis showed that subjective health status (AUC $=0.625,95 \% \mathrm{CI}: 0.594-0.656, \mathrm{P}<0.001)$ was the best predictor of vaccination behavior. Logistic regression analysis with subjective health status as a dependent variable indicated that older age, female sex, depression, neurasthenia, obsession, hypochondriasis and chronic disease were significant risk factors, while positive coping tendencies were a significant protective factor.

Conclusion. Our study found a simple and effective marker, subjective health status, that can predict vaccination behavior. This finding can guide future epidemic prevention work.

Keywords: COVID-19, vaccination behavior, vaccination rate, subjective health status, related factors 


\section{Introduction}

The significant morbidity and death rates from the COVID-19 pandemic have also caused a global economic crisis [1]. The Centers for Disease Control and Prevention (CDC) and the World Health Organization (WHO) proposed COVID-19 vaccination as an emergent and important method to end the COVID-19 pandemic [2,3]. Therefore, it has become a top priority for governments to vaccinate as many people as possible. Since China started vaccination programs in December 2020, vaccination has spread in various provinces and municipalities nationwide, and as of June 1, 2021, China reported 661,468,000 doses of COVID-19 vaccine [4]. However, this unprecedented mass vaccination also involves many difficulties in the era of the rapid dissemination of information on the internet.

To better guide vaccination programs, many studies in different periods and regions have focused on people's vaccination willingness and its related influencing factors [5-8]. For example, Wang et al. reported that among 806 nurses in Hong Kong, China, only 40.0\% intended to accept COVID-19 vaccination from 26 February to 31 March 2020 [7]. Yoda et al. conducted internet research in September 2020 in Japan and found that $65.7 \%$ of 1100 participants showed willingness to be vaccinated [8]. Another study of 3646 respondents in Bangladesh from December 2020 to January 2021 showed that $74.6 \%$ of respondents indicated their intention to be vaccinated against COVID-19 when a safe and effective vaccine was available without a fee [5]. However, to the best of our knowledge, no research has focused on vaccination behavior, which is the ultimate goal of vaccination efforts. There are too many variables in the transformation of willingness and intention into specific action; that is, even if an individual has the intention to be vaccinated against COVID-19, he or she may ultimately not be vaccinated for various reasons. Therefore, compared with vaccination willingness, the index of vaccination behavior is more intuitive, specific and practical in guiding 
vaccination programs.

At the same time, many psychosocial factors have been found to be significantly related to vaccination willingness, such as educational level [9], gender [5], age [5], residency [10], income level [11], the presence of chronic disease [5], marital status [12], subjective health status [13], mental health status [14] and coping styles [15]. Many studies have reported the vaccination willingness of patients with various chronic diseases. For example, $76.7 \%$ of 706 people in the United States living with multiple sclerosis were willing to be vaccinated [16]. Another study showed that $80.9 \%$ of Portuguese patients with multiple sclerosis were either definitely or probably willing to receive a COVID-19 vaccine [17]. A study in Bangladesh involving 506 patients with hypertension reported that $68 \%$ were willing to be vaccinated against COVID-19 [5], while the percentage of 244 diabetic patients was $61 \%$ [5]. Chan et al. reported that the intention to accept the COVID-19 vaccination among 660 cancer patients was only $17.9 \%$ [18]. From the above research, it can be seen that the vaccination intentions of patients with different chronic diseases, and even patients with the same disease, are very different. In fact, there are great differences in the severity of various chronic diseases, people's views on chronic diseases and their psychological state. Subjective health status, another indicator reflecting the health status of respondents, can integrate the above variables and shows a stable and significant correlation with vaccination willingness [13]. Therefore, subjective health status, which focuses on the actual health perceptions of individuals, should be considered and studied in vaccination programs. It is also necessary to explore the social and psychological risk factors related to subjective health status.

Confronted with these gaps in knowledge, we aimed to examine the effectiveness of various psychosocial factors in predicting vaccination behavior. Based on the literature, we hypothesized that the subjective health status of individuals has the strongest predictive power in predicting their 
vaccination behavior. We also explore the risk factors associated with psychosocial elements that have the strongest ability to predict vaccination behavior.

\section{Materials and methods}

\subsection{Study design and sample recruitment}

A cross-sectional online survey was performed among Chinese adults aged 18-65 years from 8 May to 4 June 2021, the prometaphase of the vaccination program, to obtain the rate of vaccination behavior. We collected data through Wen Juan Xing (https://www.wjx.cn/vm/YIIyx1V.aspx), a professional online questionnaire survey platform that uses anonymous self-reports.

Filtering was used to exclude data from respondents who submitted incomplete or careless responses. Due to the length of our questionnaire, it took some time to complete it. Therefore, we excluded questionnaires completed in a short time. We assumed that participants who completed the questionnaire quickly were likely to fill in the questionnaire without carefully reading and understanding the items. In addition, the questionnaire could only be submitted after all the questions had been completed, so there were no missing values in our data set. However, we also excluded some examples of obvious logical errors in forward and reverse wordings. To encourage the subjects to complete the questionnaire, we gave each participant a financial reward.

Our sample was selected by Wen Juan Xing, which retained a number of potential subjects who agreed to participate in the investigation. Wen Juan Xing contacted and selected participants in China who met the sampling quota based on gender, age, geographical region and socioeconomic status. Therefore, in our study, adult participants aged 18-65 were representative of the above demographic factors. All the data sets received were automatically uploaded to the Wen Juan Xing platform at the end of the survey. This study was approved by the ethics committee of Wengjiang District People's Hospital of Chengdu (reference number: ec-2020-002). All participants provided written informed 
consent before completing the investigation. We confirmed that all methods were performed in accordance with the relevant guidelines and regulations.

\subsection{Measures}

The respondents completed a battery of self-assessments that collected demographic information. For the Psychological Questionnaire on Emergent Public Health Events (PQEPHE) and Simple Coping Style Questionnaire (SCSQ) used in our study, we used McDonald's $\omega$ to test the internal consistency [19]. Generally, values greater than 0.90 are excellent, those in the range of $0.80-0.90$ are good, and values from $0.70-0.80$ indicate acceptable reliability. Table 1 shows that according to the current data evaluation, most of the McDonald's $\omega$ values generally had acceptable reliability.

The PQEPHE was used to evaluate the psychological status of members of the public during the pandemic outbreak. It consisted of the following five dimensions: neurasthenia (P13, P16, P17, P18, P21), depression (P3, P5, P6, P7, P8, P11), hypochondriasis (P15, P20), fear (P1, P2, P9, P12, P14) and obsession (P4, P10, P19, P22, P23, P24). Items were scored on a four-point scale ranging from 0 $=$ none to $3=$ serious; a score of 0 indicated that the respondent did not have the relevant psychological problem, while a score of 1, 2 or 3 indicated that the level of the respondent's psychological problem was mild, moderate or serious, respectively [20].

The SCSQ [21] consists of 2 dimensions, i.e., negative coping style and positive coping style, and has a total of 20 items that are scored from 0-3 each, where 0 is "not adopted" and 3 is "frequently adopted". Items 1 to 12 measure a positive coping style, and the other items measure a negative coping style. The results show the average scores of the negative coping dimension and the positive coping dimension. Previous studies have shown that the scale has good reliability and validity [22, 23].

\subsection{Statistical analysis}

Descriptive statistical statistics are shown for the sample demographics. For continuous 
variables, the mean and standard deviation were reported, and for categorical variables, the percentage was reported. Each variable was divided into vaccinated and nonvaccinated groups for comparison. The chi-square test was used for categorical variables with expected counts greater than 5, and Fisher's exact test was used for variables with expected counts less than 5. Independent-sample t-tests were used to compare the differences in continuous variables.

Next, univariate analysis was performed with demographic variables and the outcome variable to examine the association between demographic variables and vaccination behavior. Then, receiver operator characteristic (ROC) analysis was conducted to evaluate the predictive ability of all factors with significant differences and associations between the vaccinated and nonvaccinated groups as estimated by the area under the curve (AUC). Finally, ordinal multiclassification logistic regression model analysis was performed to examine the association of sociodemographic variables and mental state with the strongest predictor of vaccination behavior obtained by ROC analysis. For all analyses, the significance level was set at $p<0.05$. Statistical Product and Service Solutions software version 23.0 (SPSS Science, Chicago, IL, USA) was used for all the analyses.

\section{Results}

Of the 1300 respondents, $761(58.5 \%)$ were vaccinated, 624 (48\%) were female participants, and the average age was 30.1 years. The average education level of vaccinated respondents was 15.9 years, and that of nonvaccinated respondents was 15.5 years; there was a significant difference between the two groups $(\mathrm{p}<0.001)$. The subjective health status of vaccinated participants was significantly better than that of nonvaccinated participants $(\mathrm{p}<0.001)$. The same trend was also found for chronic diseases $(p<0.001)$, age $(p=0.026)$, positive coping tendency $(p=0.014)$, negative coping tendency $(\mathrm{p}=0.014)$, depression $(\mathrm{p}=0.001)$, neurasthenia $(\mathrm{p}<0.001)$ and obesity and anxiety $(p=0.014)$. The details of the above outcomes, marital status, income and location of residence are 
shown in Table 1.

Next, univariate analysis indicated that educational attainment at a high level was associated with higher vaccination behavior (crude odds ratio $(\mathrm{COR})=0.88,95 \% \mathrm{CI}: 0.82-0.96, \mathrm{P}=0.002$ ). Respondents who reported the presence of chronic disease were significantly $(\mathrm{COR}=1.56,95 \% \mathrm{CI}$ : $1.02-2.39, \mathrm{P}=0.041)$ more likely to decide not to be vaccinated. At the same time, the subjective health status level of respondents was a positive factor for vaccination behavior (COR $=0.62,95 \%$ CI: $0.04-1.26, \mathrm{P}=0.015)$; that is, the better the subjective health status of individuals, the more likely they were to have completed vaccination. The details of the univariate analysis are shown in Table 2.

ROC analysis showed that subjective health status was the strongest predictor of vaccination behavior among all factors $(\mathrm{AUC}=0.625,95 \% \mathrm{CI}$ : $0.594-0.656, \mathrm{P}<0.001)$. The predictive ability of other factors with significant differences between the vaccinated and nonvaccinated groups was as follows: age $(\mathrm{AUC}=0.472,95 \% \mathrm{CI}: 0.440-0.504, \mathrm{P}=0.083)$, education $(\mathrm{AUC}=0.544,95 \% \mathrm{CI}$ : 0.513-0.576, $\mathrm{P}=0.006)$, chronic diseases $(\mathrm{AUC}=0.527,95 \% \mathrm{CI}: 0.495-0.559, \mathrm{P}=0.097)$, positive coping tendency $(\mathrm{AUC}=0.541,95 \% \mathrm{CI}: 0.509-0.572, \mathrm{P}=0.013)$, negative coping tendency $(\mathrm{AUC}=0.460,95 \%$ CI: $0.428-0.492, \mathrm{P}=0.014)$, depression $\quad(\mathrm{AUC}=0.442,95 \%$ CI: $0.411-$ 0.474, $\mathrm{P}<0.001)$, neurasthenia $(\mathrm{AUC}=0.430,95 \% \mathrm{CI}: 0.399-0.461, \mathrm{P}<0.001)$ and obsession and anxiety $(\mathrm{AUC}=0.448,95 \% \mathrm{CI}: 0.416-0.480, \mathrm{P}=0.001)$. Figure 1 shows the ROC curve for the ability of all significant elements to predict vaccination behavior.

Furthermore, ordinal multiclassification logistic regression model analysis showed that age (OR: 1.05, 95\% CI: 1.03-1.06), gender (male vs. female; OR: 1.35, 95\% CI: 1.09-1.68), positive coping tendency (OR: 0.57, 95\% CI: 0.43-0.75), depression (OR: 1.56, 95\% CI: 1.14-2.15), neurasthenia (OR: 1.53, 95\% CI: 1.13-2.15), obsession and anxiety (OR: 1.59, 95\% CI: 1.38-1.91), hypochondriasis (OR: 1.75, 95\% CI: 1.59-1.96) and chronic condition (yes vs. no; OR: 0.27, 95\% 
CI: $0.18-0.40)$ were significant elements associated with concerns about subjective health status (Table 3).

\section{Discussion}

To the best of our knowledge, this is the first study on COVID-19 to take vaccination behavior as a primary outcome index and analyze its predictors. The survey was performed during May and June 2021, at a time when vaccination projects were actively promoted across China. From our online survey, we found that $58.5 \%$ of respondents had been vaccinated, and there were significant differences in subjective health status, age, education level, chronic diseases, positive coping tendency score, negative coping tendency score, depression, neurasthenia and obesity between vaccinated and nonvaccinated people. In addition, a high education level and good subjective health status were protective factors for vaccination behavior, while suffering from chronic diseases was a risk factor. ROC analysis showed that subjective health status was the best predictor of vaccination behavior among all factors. Ordinal multiclassification logistic regression analysis with subjective health status as a dependent variable indicated that older age, female sex, depression, neurasthenia, obsession, hypochondriasis and chronic disease were significant risk factors and that positive coping tendency was a significant protective factor.

Prior to novel COVID-19 SARS-CoV-2, studies generally showed that factors such as worse self-reported health, suffering from chronic diseases and older age were protective factors for the vaccination rate [24]. The same trend was observed in the initial outbreak of COVID-19 and the initial stage of vaccination $[8,13,25]$, but the trend gradually changed with the vaccination program in various countries. With the development of COVID-19 vaccination work, the vaccination intention of people with chronic diseases has become not significantly different from that of healthy people [26]. Even in elderly people, vaccination intention has become significantly lower than among 
younger people [5]. Our study shows that the prevalence of chronic diseases in the nonvaccinated population is significantly higher than that in the vaccinated population, and univariate analysis indicates that suffering from chronic disease is a risk factor for vaccination behavior. Vaccination against COVID-19 has been considered to be one of the most promising and cost-effective health interventions for the prevention and control of the pandemic. The vaccination situation of the COVID19 vaccine has been widely reported by the mass media and has attracted a high degree of attention in the population. Therefore, any possible negative news about vaccination is amplified [27], which naturally includes news of serious side effects after vaccination for patients with chronic diseases [28, 29]. A recent longitudinal study also showed that people's concerns about the side effects and ineffectiveness of vaccination increased significantly over time [26]. This may be the reason for the decreasing vaccination rate for patients with chronic diseases. In addition, our research indicated that in terms of the current vaccination rate, vaccinated people are significantly older than nonvaccinated people, which may indicate the cumulative effect of the high vaccination behavior of elderly people previously. Even if the vaccination intention of elderly people is gradually decreasing, their vaccination rate is still higher than that of the lower age group.

Consistent with previous studies, the psychological status of respondents, such as coping tendency, depression, neurasthenia and obesity, significantly affects vaccination willingness $[15,30]$. In this study, the scores for negative coping tendency, depression, neurasthenia and obesity among the vaccinated were significantly lower than those of nonvaccinated people, and the score of positive coping tendency was significantly higher among vaccinated people than among the nonvaccinated. From the perspective of cognitive behavior theory, individual cognition directly affects all types of decisions, and psychological status is the direct response of individual cognitive thinking. Therefore, individuals with high scores for negative coping tendency, depression, anxiety and hypochondriasis 
will hesitate or even refuse to be vaccinated, while those with high scores for a positive coping style are more likely to be vaccinated.

Subjective health status is an individual's subjective evaluation and expectation of his or her health status. It has become one of the common health measurement methods in the world [24]. The evaluation method adopted in our study had only one question, and the answer was a simple 5-level evaluation. This enabled us to reduce the information acquisition time to the greatest extent and intuitively obtain the health status of individual subjective feelings. Our research showed that good subjective health status was a protective factor against vaccination behavior, and ROC analysis showed that subjective health status was the best index to predict the effectiveness of vaccination behavior among all the factors, with significant differences between the vaccinated and nonvaccinated groups. This may be due to the widespread negative information about the vaccine among the population with the commencement of vaccination. Because of this, people with chronic diseases may worry that vaccination will lead to disease recurrence or induce new diseases. On the other hand, people with poor psychological status are more likely to hesitate when facing the choice of vaccination. Poor quality of life may also make people unable to find time to vaccinate. The index of subjective health status can reflect not only the status of physical diseases but also the psychological state and quality of life. It is a comprehensive evaluation index [31]. This may contribute to its unique advantage in predicting vaccination behavior. Our research focused on the final goal of current public health behaviors, vaccination behavior, and found a simple and rapid prediction index that provides a simple and reliable marker for guiding vaccination work in the current context and intervention for people who refuse or hesitate to vaccinate.

Logistic regression analysis showed that objective health status, that is, suffering from chronic diseases, was a risk factor for subjective health status, similar to previous studies [32]. Females and 
elderly people are also more likely to give pessimistic evaluations when evaluating their subjective health status than males and young people. The physical functions of elderly individuals are lower than those of young individuals to varying degrees. Due to social culture, women are more inclined to provide negative evaluations of their own health situation [33]. Negative mental states such as depression, neurasthenia, obsession and hypochondriasis can also have a negative impact on subjective health. Based on the medical concept of integrating body and mind, psychological state is an important factor to evaluate individual health status. Moreover, people with a poor psychological state are more likely to practice negative thinking, which leads to the deterioration of their self-rated health status. Similarly, a positive coping style is beneficial to the estimation of individual health status.

It is worth mentioning that education is a protective factor for vaccination willingness or vaccination behavior at any time. Studies conducted before the outbreak of COVID-19 [24], studies on the COVID-19 outbreak [13], and studies of COVID-19 vaccination [8] have begun to show that education is beneficial to increasing the vaccination rate. Therefore, education can increase the vaccination rate at any time. Although the education level of all people cannot be significantly improved in the short term, in the long run, improving the education level of all people plays a vital role in the prevention and intervention of epidemic diseases.

The limitations of this study should be considered. First, the decision to vaccinate was provided by the respondents themselves, and the reliability of the data cannot be verified. Currently, the vaccination information of all citizens in China can be easily obtained through WeChat. In future research, respondents could be required to attach screenshots of vaccination information to ensure the reliability of the data. In addition, our study was a cross-sectional study. Therefore, we cannot obtain information about the dynamic changes between subjective health status and vaccination behavior 
with the change in the vaccination form, and we cannot determine causal relationships. Furthermore, the questionnaire was designed to be simple and easy to answer, so we could not evaluate other sociodemographic factors, such as personality, sleep status and daily habits.

\section{Conclusions}

In summary, we found that $58.5 \%$ of respondents had been vaccinated before 4 June 2021 . Moreover, our study found a simple and effective marker, subjective health status, that can predict vaccination behavior, which is beneficial to guide future epidemic prevention work. Suffering from chronic diseases, female sex, elderly age and negative emotional state were significant risk factors for subjective health status, which provides direction for the next specific intervention measures. In the future, longitudinal research should continue to pay attention to the relationship between subjective health status and vaccination behavior because people receive different information in different periods, which may affect their judgment of the pros and cons of their own health status and whether they need vaccination. 


\section{Ethics approval and consent to participate}

The current study was approved by the Ethics Committee of the Wengjiang District People's Hospital of Chengdu, (reference number: ec-2020-002) China. All participants provided written informed consent.

\section{Consent for publication}

Not applicable.

\section{Competing interests}

The authors have no conflict of interest to declare.

\section{Funding}

This study was supported by research grants from the Innovation and Entrepreneurship Project of Sichuan Provincial Science and Technology department (Grand code 2020JDRC0135), National Key Research and Development Plan (grant number 2017YFC0113907), the Non-profit Central Research Institute Fund of Chinese Academy of Medical Sciences (2019PT310020) and Establishment, evaluation system construction and promotion of group model of psychosomatic nursing happiness perception training in general hospital (2018ZX04).

\section{Authors' Contributions}

Zuxing Wang, Lili Chen and Mengsha Qi had full access to the data in the study and takes responsibility for the integrity of the data and the accuracy of the data analysis. Concept and design: All authors. Acquisition, analysis, or interpretation of data: Zuxing Wang, Lili Chen and Mengsha Qi. Drafting of the manuscript: All authors. Administrative, technical, or material support: All authors. Supervision: All authors.

\section{Acknowledgement}

None.

\section{Availability of data and materials}


The datasets used and analyzed in this study are available from the corresponding author on reasonable request. 


\section{References}

1. Ataguba JE. COVID-19 Pandemic, a War to be Won: Understanding its Economic Implications for Africa. Appl Health Econ Health Policy. 2020;18(3):325-8.

2. Nguyen KH, Srivastav A, Razzaghi H, et al. COVID-19 vaccination intent, perceptions, and reasons for not vaccinating among groups prioritized for early vaccination - United States, September and December 2020. Am J Transplant. 2021;21(4):1650-6.

3. Organization WH. Coronavirus disease (COVID-19): Herd immunity, lockdowns, and COVID-19. Am J Transplant. 2021;21(4):1650-6.

4. China NHCotPRo. COVID-19 vaccination. National Health Commission of the People's Republic of China. 2021.

5. Abedin M, Islam MA, Rahman FN, et al. Willingness to vaccinate against COVID-19 among Bangladeshi adults: Understanding the strategies to optimize vaccination coverage. PLoS One. 2021;16(4):e0250495.

6. Wake AD. The Willingness to Receive COVID-19 Vaccine and Its Associated Factors: "Vaccination Refusal Could Prolong the War of This Pandemic" - A Systematic Review. Risk Manag Healthc Policy. 2021;14:2609-23.

7. Wang K, Wong E, Ho KF, et al. Intention of nurses to accept coronavirus disease 2019 vaccination and change of intention to accept seasonal influenza vaccination during the coronavirus disease 2019 pandemic: A cross-sectional survey. Vaccine. 2020;38(45):7049-56.

8. Yoda T, Katsuyama H. Willingness to Receive COVID-19 Vaccination in Japan. Vaccines (Basel). 2021;9(1).

9. Ehde DM, Roberts MK, Herring TE, Alschuler KN. Willingness to obtain COVID-19 vaccination in adults with multiple sclerosis in the United States. Mult Scler Relat Disord. 2021;49:102788.

10. Urrunaga-Pastor D, Bendezu-Quispe G, Herrera-Añazco P, et al. Cross-sectional analysis of COVID-19 vaccine intention, perceptions and hesitancy across Latin America and the Caribbean. Travel Med Infect Dis. 2021;41:102059.

11. Machida M, Nakamura I, Kojima T, et al. Acceptance of a COVID-19 Vaccine in Japan during the COVID-19 Pandemic. Vaccines (Basel). 2021;9(3).

12. Al-Mohaithef M, Padhi BK. Determinants of COVID-19 Vaccine Acceptance in Saudi Arabia: A Web-Based National Survey. J Multidiscip Healthc. 2020;13:1657-63.

13. Wong M, Wong E, Huang J, et al. Acceptance of the COVID-19 vaccine based on the health belief model: A population-based survey in Hong Kong. Vaccine. 2021;39(7):1148-56.

14. Thapa SB, Mainali A, Schwank SE, Acharya G. Maternal mental health in the time of the COVID-19 pandemic. Acta Obstet Gynecol Scand. 2020;99(7):817-8.

15. Taylor S, Landry CA, Paluszek MM, Rachor GS, Asmundson G. Worry, avoidance, and coping during the COVID-19 pandemic: A comprehensive network analysis. J Anxiety Disord. 2020;76:102327.

16. Uhr L, Mateen FJ. COVID-19 vaccine hesitancy in multiple sclerosis: A cross-sectional survey. Mult Scler. 2021:13524585211030647.

17. Serrazina F, Sobral Pinho A, Cabral G, Salavisa M, Correia AS. Willingness to be vaccinated against COVID-19: An exploratory online survey in a Portuguese cohort of multiple sclerosis patients. Mult Scler Relat Disord. 2021;51:102880.

18. Chan WL, Ho YT, Wong CK, et al. Acceptance of COVID-19 Vaccination in Cancer Patients in Hong Kong: Approaches to Improve the Vaccination Rate. Vaccines (Basel). 2021;9(7).

19. McNeish D. Thanks coefficient alpha, we'll take it from here. Psychol Methods. 2018;23(3):412-33.

20. Qi M, Li X, Liu S, Li Y, Huang W. Impact of the COVID-19 epidemic on patterns of pregnant women's perception of threat and its relationship to mental state: A latent class analysis. PLoS One. 2020;15(10):e0239697.

21. Wang L, Kang C, Yin Z, Su F. Psychological Endurance, Anxiety, and Coping Style among Journalists Engaged in Emergency Events: Evidence from China. Iran J Public Health. 2019;48(1):95-102.

22. Dai WJ, Chen L, Tan HZ, et al. [Influence of social support and coping style on chronic post-traumatic stress disorder after floods]. Zhonghua Liu Xing Bing Xue Za Zhi. 2016;37(2):214-7.

23. Xia Y, Zhang H, Xia Y, Li H, Zhai L, Wang H. The self-psychological safety maintenance and its influencing factors of 
community frontline staff during COVID-19 pandemic. Medicine (Baltimore). 2021;100(3):e24140.

24. Zürcher K, Zwahlen M, Berlin C, Egger M, Fenner L. Losing ground at the wrong time: trends in self-reported influenza vaccination uptake in Switzerland, Swiss Health Survey 2007-2017. BMJ Open. 2021;11(2):e041354.

25. Seale H, Heywood AE, Leask J, et al. Examining Australian public perceptions and behaviors towards a future COVID-19 vaccine. BMC Infect Dis. 2021;21(1):120.

26. Babicki M, Mastalerz-Migas A. Attitudes toward Vaccination against COVID-19 in Poland. A Longitudinal Study Performed before and Two Months after the Commencement of the Population Vaccination Programme in Poland. Vaccines (Basel). 2021;9(5).

27. Wan E, Chui C, Lai F, et al. Bell's palsy following vaccination with mRNA (BNT162b2) and inactivated (CoronaVac) SARSCoV-2 vaccines: a case series and nested case-control study. Lancet Infect Dis. 2021.

28. Dai C, Jiang M, Huang YH. COVID-19 Vaccination in Patients with Inflammatory Bowel Disease: A Survey from China. Dig Dis Sci. 2021.

29. Verma AK, Lavine KJ, Lin CY. Myocarditis after Covid-19 mRNA Vaccination. N Engl J Med. 2021.

30. Palgi Y, Bergman YS, Ben-David B, Bodner E. No psychological vaccination: Vaccine hesitancy is associated with negative psychiatric outcomes among Israelis who received COVID-19 vaccination. J Affect Disord. 2021;287:352-3.

31. Pasanen TP, Tamminen N, Martelin T, Mankinen K, Solin P. Profiles of subjective health among people living alone: a latent class analysis. BMC Public Health. 2021;21(1):1335.

32. Chow S, Lam KM, Lie SH, et al. Do demographic factors and a health-promoting lifestyle influence the self-rated health of college nursing students. BMC Nurs. 2018;17:50.

33. Carvalho de Abreu DC, Freire Junior RC, Mello Porto J, Errera Magnani P, Silva Tofani P, Rodrigues Iosimuta NC. Functional performance of older adults: A comparison between men and women. Women Health. 2021;61(4):372-80. 

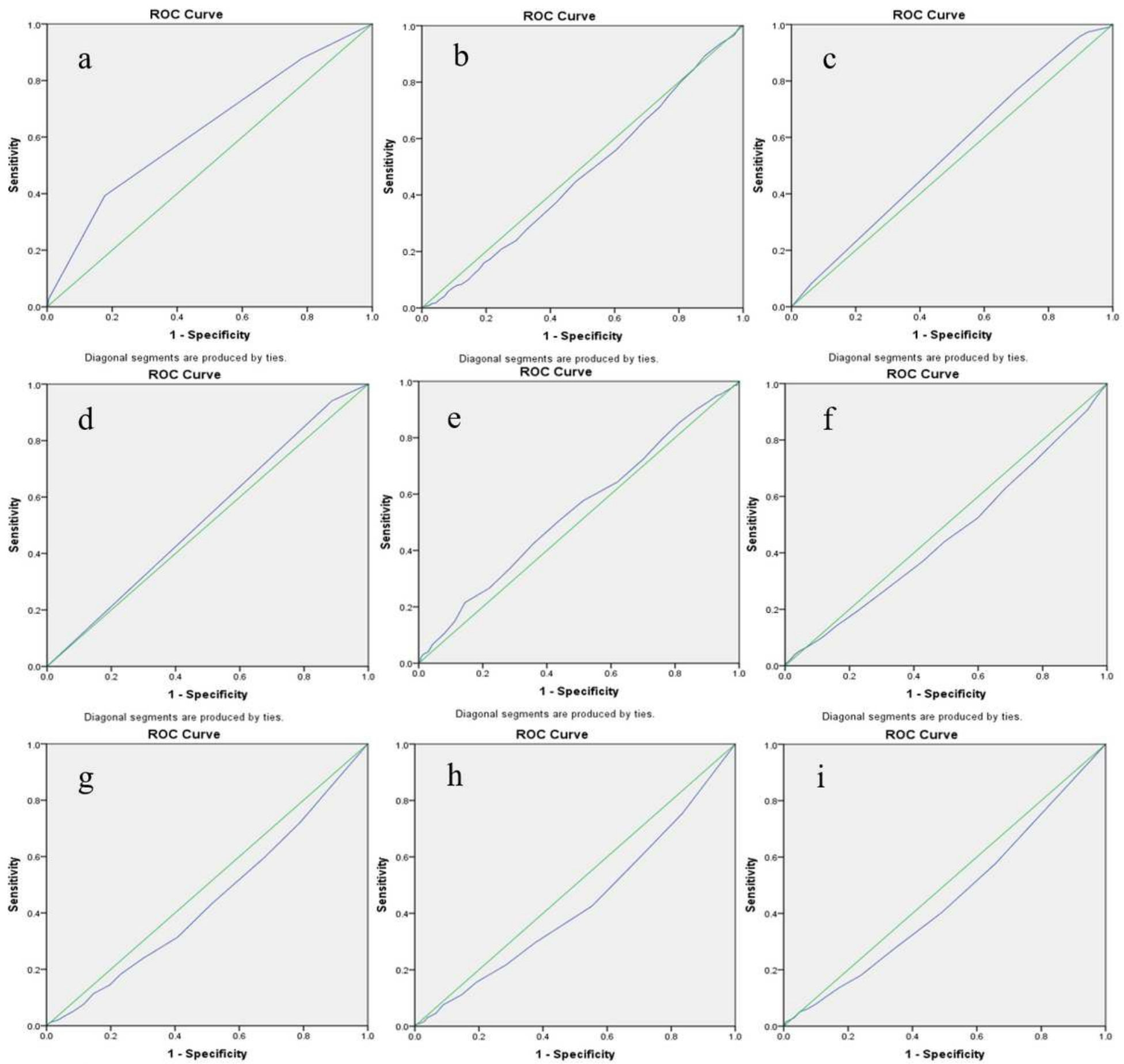

Diagonal segments are produced by ties.

Diagonal segments are produced by ties.

Diagonal segments are produced by ties.

Figure 1

ROC curve of significant elements in predicting the vaccination behavior a. ROC curve of subjective health status for predicting vaccination behavior, $b$. ROC curve of age for predicting vaccination behavior, $c$. ROC curve of education for predicting vaccination behavior, $d$. ROC curve of chronic diseases for predicting vaccination behavior, e. ROC curve of positive coping tendency for predicting vaccination behavior, $\mathrm{f}$. ROC curve of negative coping tendency for predicting vaccination behavior, g. ROC curve of depression for 
predicting vaccination behavior, h. ROC curve of neurasthenia for predicting vaccination behavior, i. ROC curve of obsession and anxiety for predicting vaccination behavior. 